\title{
Predictability of Vesicoureteral Reflux Using Interlobar Renal Arterial Resistive Indices in Sonographically Normal Pediatric Kidneys-Preliminary Results
}

\author{
John Amodio ${ }^{*}$, Rafael Rivera ${ }^{2}$, Lynne Pinkney ${ }^{2}$, Naomi Strubel ${ }^{2}$, Nancy Fefferman ${ }^{2}$ \\ ${ }^{1}$ Department of Radiology, SUNY Downstate Medical Center, New York, USA \\ ${ }^{2}$ Department of Radiology, New York University School of Medicine, New York, USA \\ Email: *John.Amodio@Downstate.edu
}

Received May 17, 2012; revised June 20, 2012; accepted June 30, 2012

\begin{abstract}
Objective: To prospectively evaluate the diagnostic utility of interlobar renal arterial resistive index values, in predicting the presence of vesicoureteral reflux in children with sonographically normal appearing kidneys. Methods: We investigated 35 children with a history of urinary tract infection with grayscale and color Doppler ultrasound (US) followed by a standard voiding cystouretherogram to assess the presence of vesicoureteral reflux. All renal units (individual kidneys) were morphologically normal without evidence of hydronephrosis. Resistive index (RI) was measured at the level of the interlobar arteries. Results: Of a total of 67 kidney units, 3 kidney units yielded no diagnostic results due to technical factors (crying, agitation). 46 kidney units (69\%) were not associated with any degree of reflux. 17 kidney units (25\%) were associated with low-mid grade reflux (grades I - III). 4 kidney units (6\%) were associated with high grade reflux (grades IV - V). No statistically significant correlation was found between the interlobar resistive indices of sonographically normal appearing kidneys and the presence, absence, or degree of vesicoureteral reflux. Conclusions: The results of this study suggest that resistive index measurements taken in the interlobar arteries of sonographically normal appearing kidneys cannot be used to predict the presence or absence of vesicoureteral reflux.
\end{abstract}

Keywords: Kidney; Reflux; Resistive Index; Ultrasound; Urinary Tract Infection; Pediatrics

\section{Introduction}

Urinary tract infections are very common in the pediatric population, especially in girls. Most children with vesicoureteral reflux and urinary tract infections have an excellent prognosis with resolution of reflux by 4 years of age. The greatest risk of renal involvement in urinary tract infection is in newborn boys and pre-school age girls [1].

According to the guidelines of the American Academy of Pediatrics (AAP), a sonogram should be performed on all infants and young children with fever and their first documented UTI; prior to 2011, according to the AAP guidelines of 1999, voiding cystourethrography (VCUG) or radionuclide cystography (RCN) were recommended. New AAP guidelines [2] have recently been proposed for children with urinary tract infections (UTI). It is now recommended that VCUG or RCN not be done after the first febrile UTI. This is based on the fact that "the risks, costs, and discomfort of the VCUG are hard to justify, because there is no evidence that patients benefit from

${ }^{*}$ Corresponding author. having their VUR diagnosed" [2].

Regardless of the new AAP recommendations, it has been shown that a normal renal sonogram does not exclude the presence of vesicoureteral reflux, even in children aged 5 years or older. Therefore, if reflux is considered, a VCUG must be performed even in older children, regardless of the sonographic findings [3].

There are some studies regarding the effects of reflux on renal blood flow. Paltiel [4] studied the effect of chronic, low pressure reflux on renal growth and function in a porcine model. The results of that study showed that such reflux, in pigs, led to mild chronic interstitial inflammation and fibrosis after 1 year. However, the imaging findings were normal.

Helin [5] studied blood flow parameters, including renal vascular resistance, in piglets with unilateral ureteric pressure elevation, as may happen in vesicouretero reflux. In that study no significant changes in renal vascular resistance was found between normal kidneys and those in which there was elevated ureteric pressure.

Resistive index (RI) is defined, in Doppler ultrasound, as the ratio of the peak systolic velocity minus the end 
diastolic velocity/peak systolic velocity. RI is widely used a measurement of resistance of arterial flow, and eliminates the need for precise angle-measurements of blood flow. Most ultrasound machines can automatically calculate the RI within an arterial structure automatically, as illustrated in Figure 1.

Radmayr [6] studied the relationship for resistive index (RI), reflux and scarring. Patients with VUR grades IV and V demonstrated a statistically significant increase in RI compared to those with low grade or no reflux. The author concluded that a possible cause of these elevated RIs may be the presence of renal scarring, found in many children with higher grades of reflux. The author also recommended routine determination of RI during renal ultrasound examinations to detect renal injury and scarring in patients with VUR.

Our study was performed in effort to evaluate the following research questions:

1) Is there an alteration of the resistive indices of the interlobar arteries, in children with reflux and sonographically normal appearing kidneys?

2) If such an alteration in RI exists, can these measurements be used to predict the presence or absence of vesicoureteral reflux?

3) Is there a correlation between resistive index and the grade of reflux?

Given the known pathophysiology of reflux and renal blood flow in animals with reflux, our hypothesis was that resistive index would not be a positive predictor of reflux in children, and therefore, not a useful tool to predict the presence or absence of reflux in normal appearing kidneys on sonography.

\section{Materials and Methods}

After obtaining IRB approval, we investigated 35 children with a history of UTI with grayscale and color Doppler US followed by a standard voiding cystouretherogram to assess the presence of vesicoureteral reflux. Ages ranged from newborn - 12 years of age. There were 14 males and 21 females. All renal units (individual kidneys) were morphologically normal without evidence of hydronephrosis or renal scarring. Resistive index (RI) was measured at the level of the interlobar arteries. The arcuate arteries were not selected due to technical difficulties in obtaining Doppler measurements in such small structures, which relies on patient cooperation. Three measurements of the interlobar RIs were obtained at the level of the upper, mid, and lower poles of each kidney.

All sonograms were performed on the Siemens Antares unit. Each sonogram was performed by one of two experienced ultrasound technicians. All VCUGs were performed fluoroscopically and the results of each study were tabulated by one of five experienced pediatric radiologists (with a range of 5 - 20 years of experience). Reflux grade was assigned according to the international reflux grading system (Grades I - V). RI values were then

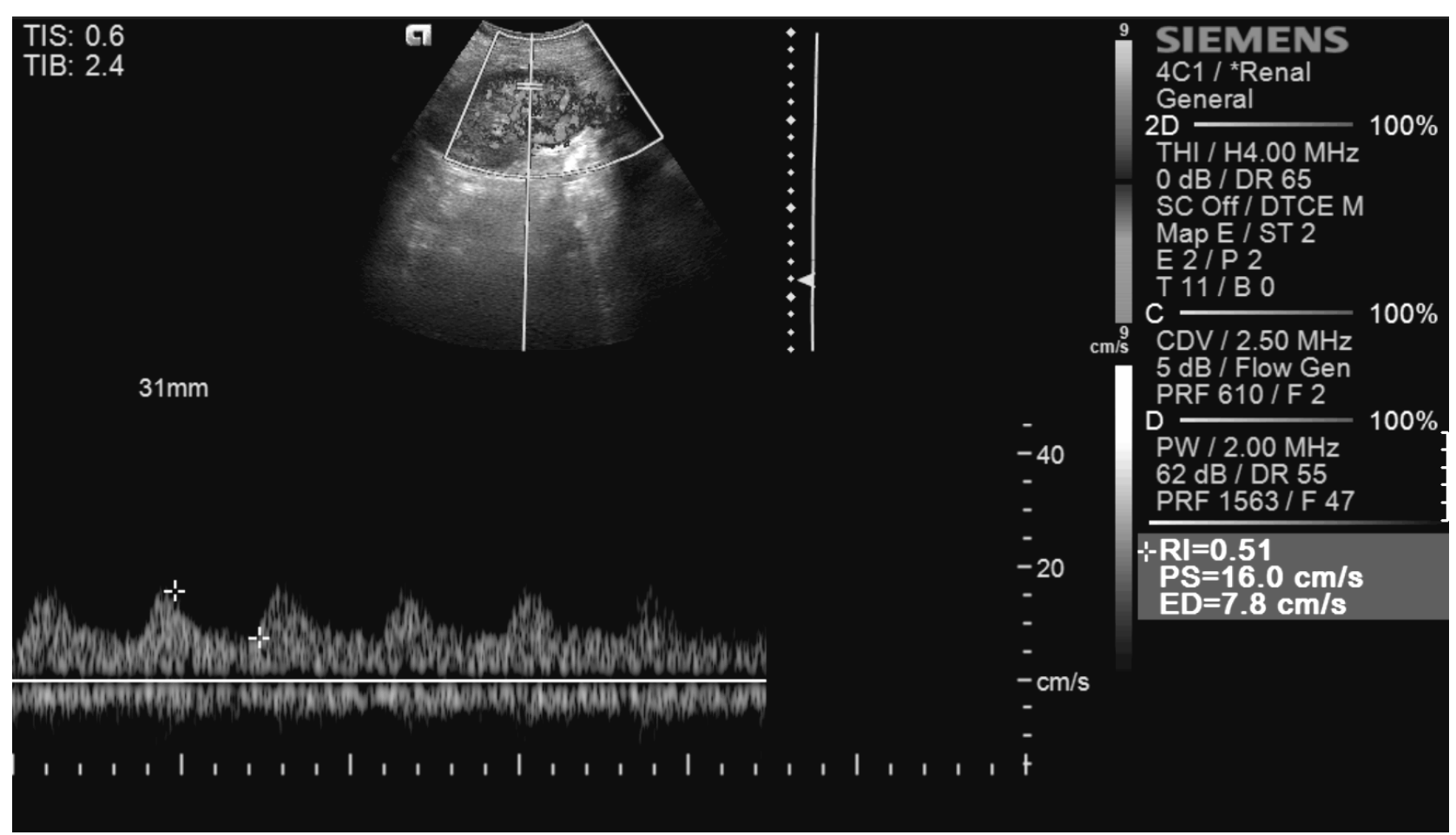

Figure 1. Spectral Doppler ultrasound of the interlobar arteries of the kidney, demonstrating the calculation of the resistive index. In this case, the RI is 0.51 . 
tabulated with VCUG results.

\section{Results}

A tabulation of the results of this study is presented in Table 1. A total of 67 kidney units were assessed. 3 kidney units yielded no diagnostic results due to technical factors (crying, agitation). 46 kidney units (69\%) were not associated with any degree of reflux. 17 kidney units
(25\%) were associated with low-mid grade reflux (grades I - III). 4 kidney units (6\%) were associated with high grade reflux (grades IV - V).

The mean RI values obtained in the three positions of each kidney are summarized in Table 2. The overall mean RI was 0.68 .

Table 3 demonstrates the number and percentages of infants with each grade of reflux in both kidneys. Here,

Table 1. Summary of RI values and presence, absence, and grade of reflux.

\begin{tabular}{|c|c|c|c|c|c|c|c|c|c|c|}
\hline Patient & Sex & Age & Rt kid up & Rt kid mid & Rt kid low & Lt kid up & Lt kid mid & Lt kid low & Reflux rt & Reflux lt \\
\hline 1 & M & 4 weeks & 0.72 & 0.74 & 0.62 & 0.69 & 0.7 & 0.72 & NO & NO \\
\hline 3 & $\mathrm{~F}$ & 624 weeks & 0.66 & 0.57 & 0.68 & 0.63 & 0.6 & 0.67 & NO & NO \\
\hline 4 & $\mathrm{~F}$ & 8 weeks & 0.6 & 0.63 & 0.67 & 0.69 & 0.63 & 0.65 & NO & NO \\
\hline 6 & M & 44 weeks & 0.74 & 0.73 & & 0.74 & 0.66 & & Grade 2 & Grade 5 \\
\hline 7 & $\mathrm{~F}$ & 104 weeks & 0.65 & 0.65 & 0.65 & 0.73 & 0.65 & 0.73 & NO & NO \\
\hline 8 & M & 0 week & $0.61,0.6$ & 0.61 & 0.58 & 0.6 & 0.67 & 0.62 & Grade 5 & Grade 5 \\
\hline 9 & M & 20 weeks & 0.84 & 0.85 & 0.83 & 0.87 & 0.8 & 0.84 & NO & NO \\
\hline 10 & $\mathrm{~F}$ & 72 weeks & 0.73 & 0.64 & 0.69 & $\mathrm{~N} / \mathrm{A}$ & N/A & $\mathrm{N} / \mathrm{A}$ & NO & Grade 4 \\
\hline 12 & $\mathrm{~F}$ & 364 weeks & 0.78 & 0.78 & 0.62 & 0.74 & 0.7 & 0.7 & $\mathrm{NO}$ & NO \\
\hline 13 & $\mathrm{~F}$ & 104 weeks & 0.69 & 0.75 & 0.71 & 0.64 & 0.67 & 0.59 & $\mathrm{NO}$ & Grade 1 \\
\hline 14 & $\mathrm{~F}$ & 56 weeks & 0.7 & 0.65 & 0.65 & \multicolumn{3}{|c|}{ Absent left kidney } & Grade 5 & NO \\
\hline 15 & M & 28 weeks & 0.81 & 0.82 & 0.79 & 0.8 & 0.78 & 0.79 & NO & $\mathrm{NO}$ \\
\hline 16 & $\mathrm{~F}$ & 6 weeks & 0.69 & 0.55 & 0.64 & 0.68 & 0.75 & 0.72 & NO & $\mathrm{NO}$ \\
\hline 17 & M & 78 weeks & 0.75 & 0.77 & 0.77 & 0.75 & 0.75 & 0.74 & $\mathrm{NO}$ & Grade 2 \\
\hline 18 & $\mathrm{~F}$ & 60 weeks & 0.6 & 0.68 & 0.72 & 0.7 & 0.75 & 0.58 & Grade 2 & Grade 3 \\
\hline 19 & $\mathrm{~F}$ & 208 weeks & 0.61 & 0.69 & 0.71 & 0.58 & 0.67 & 0.72 & Grade 2 & \\
\hline 20 & M & 244 weeks & 0.69 & 0.68 & 0.73 & 0.57 & 0.58 & 0.67 & Grade 3 & NO \\
\hline 21 & $\mathrm{~F}$ & 260 weeks & N/A & $\mathrm{N} / \mathrm{A}$ & N/A & 0.58 & 0.67 & 0.61 & Grade 3 & Grade 3 \\
\hline 23 & M & 156 weeks & 0.76 & 0.75 & 0.74 & 0.71 & 0.59 & 0.58 & NO & $\mathrm{NO}$ \\
\hline 24 & $\mathrm{~F}$ & 130 weeks & 0.69 & 0.62 & 0.64 & 0.68 & 0.62 & 0.66 & $\mathrm{NO}$ & Grade 2-3 \\
\hline 25 & $\mathrm{~F}$ & 36 weeks & 0.67 & 0.77 & 0.69 & 0.68 & 0.66 & 0.72 & $\mathrm{NO}$ & $\mathrm{NO}$ \\
\hline 26 & $\mathrm{~F}$ & 12 weeks & 0.63 & 0.63 & 0.54 & 0.67 & 0.72 & 0.8 & NO & $\mathrm{NO}$ \\
\hline 27 & M & 4 weeks & 0.75 & 0.79 & 0.69 & 0.74 & 0.81 & 0.75 & $\mathrm{NO}$ & NO \\
\hline 28 & $\mathrm{~F}$ & 572 weeks & 0.59 & 0.61 & 0.56 & 0.65 & 0.69 & 0.56 & NO & $\mathrm{NO}$ \\
\hline 29 & $\mathrm{~F}$ & 468 weeks & 0.6 & 0.53 & 0.59 & 0.63 & 0.79 & 0.57 & Grade3 & NO \\
\hline 30 & M & 0 week & 0.67 & 0.48 & 0.63 & 0.68 & 0.7 & 0.71 & Grade 2 & Grade 3 \\
\hline 31 & M & 44 weeks & 0.73 & 0.76 & 0.74 & 0.65 & 0.71 & 0.62 & NO & $\mathrm{NO}$ \\
\hline 32 & M & 8 weeks & 0.72 & 0.74 & 0.69 & 0.7 & 0.72 & 0.7 & NO & $\mathrm{NO}$ \\
\hline 33 & $\mathrm{~F}$ & 36 weeks & 0.67 & 0.77 & 0.69 & 0.68 & 0.66 & 0.72 & NO & $\mathrm{NO}$ \\
\hline 34 & $\mathrm{~F}$ & 45 weeks & 0.57 & 0.62 & 0.74 & 0.53 & 0.52 & N/A & NO & NO \\
\hline 35 & M & 32 weeks & 0.71 & N/A & N/A & N/A & Absent l & ft kidney & Grade 3 & N/A \\
\hline
\end{tabular}


$67.65 \%$ of the sample size's right kidneys demonstrated no reflux, and $69.70 \%$ of the study population's left kidneys demonstrated no reflux.

Each p value in Table $\mathbf{4}$ is two-sided, and was not adjusted for the fact that multiple hypothesis tests were performed. Since a multiple hypothesis correction will increase each p value, and no p value is less than 0.05 , then the overall conclusion is that there are no significant results (irrespective of correction).

Our study had $80 \%$ power at the $5 \%$ significance level to detect a decline of at least 0.065 in the resistive index associated with reflux in either the left or right kidney. More specifically, a test to determine whether reflux is associated with a decline in resistive index would have approximately $80 \%$ power if the true mean of the resistive index is 0.065 units lower among infants with reflux than among those without it.

Table 2. Resistive index mean ( \pm standard deviation) of the resistive index in the upper, middle, and lower poles of the right and left kidneys.

\begin{tabular}{ccc}
\hline Position & Right kidney & Left kidney \\
\hline Lower pole & $0.679+/-0.07$ & $0.676+/-0.07$ \\
Mid pole & $0.687+/-0.09$ & $0.680+/-0.07$ \\
Upper pole & $0.688+/-0.07$ & $0.676+/-0.08$ \\
\hline
\end{tabular}

Table 3. The number and percentage of infants with each grade of reflux in the right and left kidneys.

\begin{tabular}{ccccc}
\hline \multirow{2}{*}{ Reflux grade } & \multicolumn{2}{c}{ Right kidney } & \multicolumn{2}{c}{ Left kidney } \\
\cline { 2 - 5 } & Count & Percent & Count & Percent \\
\hline 0 (none) & 23 & 67.65 & 23 & 69.70 \\
1 & 1 & 2.94 & 1 & 3.03 \\
2 & 5 & 14.71 & 1 & 3.03 \\
3 & 3 & 8.82 & 5 & 15.15 \\
4 & 0 & 0.00 & 1 & 3.03 \\
5 & 2 & 5.88 & 2 & 6.06 \\
Total & 34 & 100 & 33 & 100 \\
\hline
\end{tabular}

Table 4. $p$ values to compare infants with and without reflux in the left kidney, right kidney, and at least one kidney with respect to the resistive index in each position of the kidney.

\begin{tabular}{ccccc}
\hline Resistive index location & $\begin{array}{c}\text { Right kidney } \\
\text { reflux: absent } \\
\text { versus present }\end{array}$ & $\begin{array}{c}\text { Left kidney } \\
\text { reflux: } \\
\text { absent verses } \\
\text { present }\end{array}$ & $\begin{array}{c}\text { Reflux in } \\
\text { either } \\
\text { kidney: yes } \\
\text { vs. no }\end{array}$ \\
\hline Left & Lower & 0.084 & 0.250 & 0.085 \\
Left & Middle & 0.527 & 0.930 & 0.597 \\
Left & Upper & 0.095 & 0.948 & 0.246 \\
Right & Lower & 0.487 & 0.803 & 0.908 \\
Right & Middle & 0.095 & 0.413 & 0.140 \\
Right & Upper & 0.271 & 0.752 & 0.715 \\
\hline
\end{tabular}

Table 5 demonstrates the $\mathrm{p}$ value between the resistive index in each location and reflux grade in each kidney. Again, it was not adjusted for multiple hypothesis tests, since the uncorrected $\mathrm{p}$ values were greater than 0.05 . The conclusion is that there is no significant correlation between resistive index and reflux grade, irrespective of whether a multiple hypothesis correction is applied.

Lastly, Table 6 demonstrates the statistical significance in predicting reflux at various parts of the kidney with a given resistive index, adjusted after the potential confounding effects of age and gender. This logistical regression analysis had an $80 \%$ power at the $5 \%$ significance level to detect an odds ratio of 2.85. It, moreover, successfully concludes that there is no significant predictive value of resistive index in reflux. The left lower resistive index demonstrated a p value of less than 0.1 in predicting reflux in at least one kidney.

\section{Discussion}

What is the normal RI value of the interlobar arteries in children? Radmayr [6] found that RI values are related to age. In children less than a year of age, the maximum RI value is $0.77+/-0.08$. In the second year of life on, the RIs are less than 0.7 . The mean RI taken at all ages is 0.6 $+/-0.06$. All of the RIs obtained in our study were within this range and therefore normal.

Table 5. Spearman correlation (p value) between the resistive index in each location and the reflux grade in the kidney.

\begin{tabular}{cccc}
\hline \multicolumn{2}{c}{ Resistive index location } & \multicolumn{2}{c}{ Correlation with reflux grade in } \\
\hline Kidney & Position & Right kidney & Left kidney \\
Left & Lower & $-0.34(0.07)$ & $-0.23(0.22)$ \\
Left & Middle & $-0.09(0.62)$ & $-0.04(0.84)$ \\
Left & Upper & $-0.35(0.06)$ & $0.01(0.97)$ \\
Right & Lower & $-0.15(0.40)$ & $-0.0(0.99)$ \\
Right & Middle & $-0.32(0.07)$ & $-0.19(0.31)$ \\
Right & Upper & $-0.21(0.24)$ & $0.05(0.80)$ \\
\hline
\end{tabular}

Table 6. $p$ value assessment, from logistic regression, of predicting reflux in the right kidney, left kidney, or at least one of the two kidneys, after adjusting for infant age and gender.

\begin{tabular}{ccccc}
\hline \multicolumn{2}{c}{ Resistive index location } & \multicolumn{3}{c}{ Predicting reflux in } \\
\hline Kidney & Position & Right kidney & Left kidney & At least 1 \\
\hline Left & Lower & 0.127 & 0.194 & 0.093 \\
Left & Middle & 0.619 & 0.752 & 0.664 \\
Left & Upper & 0.120 & 0.703 & 0.217 \\
Right & Lower & 0.472 & 0.998 & 0.984 \\
Right & Middle & 0.078 & 0.308 & 0.133 \\
Right & Upper & 0.164 & 0.984 & 0.605 \\
\hline
\end{tabular}


Measurement of resistive index is a noninvasive diagnostic modality for studying changes in renovascular resistance. Since it is a ratio, and independent of the Doppler angle, RI values are an ideal way of studying intrarenal resistance in difficult or non cooperative patients. Elevated RIs (RI > 0.7) can be encountered in diseases involving the tubulointerstitial or vascular system (e.g. lupus nephritis) $[7,8]$.

It is well known that intrarenal reflux has a predilection for affecting the upper and lower poles of the kidney, which is thought to be the result of the morphology and vertical orientation of the renal papillae in these regions. [9]. Funson and Cremin [10] found that there is an abundance of compound papillae in the upper and lower pole regions and were more subject to intra renal reflux, especially at lower pressures. Therefore, might the RIs be different in the upper and lower poles compared to the mid pole region? Three regions of each kidney were studied with Doppler sonography in our study, to determine if there are any differences in RI measurements in these locations in refluxing kidneys. However, no statistically significant differences in the RI values in the various poles of the kidneys, associated with reflux alone, were seen.

Kawauchi et al. [11] studied two populations of children, and both groups demonstrated vesicoureteral reflux. One group, however, did not demonstrate renal scarring on radionuclide studies, while the other group did demonstrate evidence of renal scarring. The results of this study showed that the RIs were significantly elevated in kidneys with scars compared to those without scars. The authors concluded that it must be renal scarring which produces parenchymal damage, which in turn, elevates the RI and not reflux alone. The authors also concluded that the increased RI in refluxing kidneys may predict the presence of renal scarring and is therefore useful for follow up in patients with documented reflux on VCUG. The results of our study would suggest this may be true.

There are several limitations of our study. The sample size is small and this study is continuing to acquire more data. Given our results, a higher sample size may have theoretically produced a statistically significant association between RI and vesicoureteral reflux. While the statistical power in our study was enough to demonstrate a difference had one existed, a larger sample size may have yielded $\mathrm{p}$ values less than 0.05 .

Secondly, none of our patients had radionuclide imaging to document the presence or absence of renal scarring. We assumed that no significant parenchymal damage existed, since all of the kidneys were morphologically normal on sonography. However, nuclear imaging is more sensitive for the detection of renal scarring than ultrasound [12].

Our study, however, is a harbinger for future studies. It is the first, using sonographically normal kidneys, to investigate the predictive value of resistive indices in vesicoureteral reflux. Other studies have demonstrated the importance of each variable thus far (i.e. RI or reflux), but have not used a model of clinically normal kidneys to extract a possible relationship. Such an undertaking accurately simulates clinical scenarios. In addition, the prior literature suggests that elevated RIs are the result of parenchymal scarring. Therefore, elevated renal RIs in the face of reflux might be a predictor of renal scarring, and might prompt the investigation of such using radionuclide imaging.

In summary, our preliminary results suggest that resistive index measurements taken in the interlobar arteries of sonographically normal appearing kidneys do not predict the absence or presence of vesicoureteral reflux. Additionally, no statistically significant correlation exists between RI values and reflux grade. Our results also confirm prior studies, in that elevated RIs are not related to reflux per se, but are more likely to be a predictor of renal scarring.

In the imaging workup of children with urinary tract infections and sonographically normal appearing kidneys, interlobar RI values are not predictive of vesicoureteral reflux. A VCUG is still required to evaluate for the presence of reflux. However, elevated renal RIs may suggest renal scarring, in the presence of reflux, and lead to further work-up with radionuclide imaging.

\section{REFERENCES}

[1] G. Ozcelij, T. B. Polat, S. Aktas, et al., "Resistive Index in Febrile Urinary Tract Infections: Predictive Value of Renal Outcome,” Pediatric Nephrology, Vol. 19, No. 2, 2004, pp. 148-152. doi:10.1007/s00467-003-1305-z

[2] "Urinary Tract Infection: Clinical Practice Guideline for the Diagnosis and Management of the Initial UTI in Febrile Infants and Children 2 to 24 Months,” Pediatrics, Vol. 128, No. 3, 2011, pp. 595-610.

http://pediatrics.aappublications.org/content/128/3/595.ab stract?sid=55efde08-204d-42ff-8055-dc65999b96b2

[3] M. A. Di Pietro, C. E. Blane and J. M. Zerin, "Vesicouretral Reflux in Older Children: Concordance of US and Voiding Cystourethrographic Findings,” Radiology, Vol. 205, No. 3, 1997, pp. 821-822.

[4] H. Paltiel, R. Mulkern, A. Perez-Atayade, et al., "Effect of Chronic, Low Pressure, Sterile Vesicoureteral Reflux on Renal Growth and Function in a Porcine Model: A Radiologic and Pathologic Study,” Radiology, Vol. 217, No. 2, 2000, pp. 507-515.

[5] I. Helin, L. Okmian and T. Olin, "Renal Blood Flow and Elevated Ureteric Pressure," Scandinavian Journal of Urology and Nephrology, Vol. 28, No. 1, 1975, pp. 5369.

[6] C. Radmayr, A. Klauser, C. Maneschg, et al., "Importance of Renal Resistive Index in Children Suffering from Vesi- 
coureteral Reflux,” European Urology, Vol. 36, No. 1, 1999, pp. 75-79. doi:10.1159/000019931

[7] M. Arima, M. Ishibashi, M. Usami, et al., "Analysis of the Arterial Blood Flow Patterns of Normal and Allografted Kidneys by the Directional Ultrasonic Doppler Technique,” Journal of Urology, Vol. 122, No. 1, 1979, pp. 587-590.

[8] J. F. Platt, J. M. Rubin and J. H. Ellis, "Lupus Nephritis: Predictive Value of Conventional and Doppler US and Comparison with Serologic and Biopsy Parameters," Radiology, Vol. 203, No. 1, 1997, pp. 82-86.

[9] J. Haller, “Urinary Tract and Retroperitoneum,” In: K. Slovis and Haller, Eds., Caffey's Pediatric Diagnostic Imaging, 10th Edition, Mosby, Philadelphia, 2004, p.
1860.

[10] M. R. Funston and B. J. Cremin, "Intrarenal RefluxPapillary Morphology and Pressure Relationships in Children's Necropsy Kidneys,” British Journal of Radiology, Vol. 51, No. 609, 1978, pp. 665-670. doi:10.1259/0007-1285-51-609-665

[11] A. Kawauchi, Y. Yamao, O. Ukimura, et al., "Evaluation of Reflux Using Renal Resistive Index,” Journal of Urology, Vol. 165, No. 6, 2001, pp. 2010-2012. doi:10.1016/S0022-5347(05)66282-9

[12] A. Calado, U. Barroso, V. Barroso, et al., "Ultrasound Evaluation of Renal Scarring in Children with Vesicoureteral Reflux,” Brazilian Journal of Urology, Vol. 28, No. 3, 2002, pp. 250-253. 